\title{
New real-variable characterizations of Hardy spaces associated with twisted convolution
}

Jizheng Huang* and Zhou Xing

\section{"Correspondence:} hjzheng@163.com

College of Sciences, North China University of Technology, Beijing, 100144, P.R. China

\section{Abstract}

In this paper, we give some new real-variables characterizations of the Hardy space associated with twisted convolution, including Poisson maximal function, area integral, and Littlewood-Paley g-function.

MSC: 42B30; 42B25; 42B35

Keywords: twisted convolution; Hardy space; Poisson maximal function; area integral; Littlewood-Paley g-function

\section{Introduction}

In this paper, we consider the $2 n$ linear differential operators

$$
Z_{j}=\frac{\partial}{\partial z_{j}}+\frac{1}{4} \bar{z}_{j}, \quad \bar{Z}_{j}=\frac{\partial}{\partial \bar{z}_{j}}-\frac{1}{4} z_{j} \quad \text { on } \mathbb{C}^{n}, j=1,2, \ldots, n \text {. }
$$

Together with the identity they generate a Lie algebra $h^{n}$ which is isomorphic to the $2 n+1$ dimensional Heisenberg algebra. The only nontrivial commutation relations are

$$
\left[Z_{j}, \bar{Z}_{j}\right]=-\frac{1}{2} I, \quad j=1,2, \ldots, n
$$

The operator $L$ defined by

$$
L=-\frac{1}{2} \sum_{j=1}^{n}\left(Z_{j} \bar{Z}_{j}+\bar{Z}_{j} Z_{j}\right)
$$

is nonnegative, self-adjoint, and elliptic. Therefore it generates a diffusion semigroup $\left\{T_{t}^{L}\right\}_{t>0}=\left\{e^{-t L}\right\}_{t>0}$. The operators in (1) generate a family of 'twisted translations' $\tau_{w}$ on $\mathbb{C}^{n}$ defined on measurable functions by

$$
\begin{aligned}
\left(\tau_{w} f\right)(z) & =\exp \left(\frac{1}{2} \sum_{j=1}^{n}\left(w_{j} z_{j}+\bar{w}_{j} \bar{z}_{j}\right)\right) f(z) \\
& =f(z+w) \exp \left(\frac{i}{2} \operatorname{Im}(z \cdot \bar{w})\right)
\end{aligned}
$$

(c) 2015 Huang and Xing. This article is distributed under the terms of the Creative Commons Attribution 4.0 International License (http://creativecommons.org/licenses/by/4.0/), which permits unrestricted use, distribution, and reproduction in any medium, provided you give appropriate credit to the original author(s) and the source, provide a link to the Creative Commons license, and indicate if changes were made. 
The 'twisted convolution' of two functions $f$ and $g$ on $\mathbb{C}^{n}$ can now be defined as

$$
\begin{aligned}
(f \times g)(z) & =\int_{\mathbb{C}^{n}} f(w) \tau_{-w} g(z) d w \\
& =\int_{\mathbb{C}^{n}} f(z-w) g(w) \bar{\omega}(z, w) d w
\end{aligned}
$$

where $\omega(z, w)=\exp \left(\frac{i}{2} \operatorname{Im}(z \cdot \bar{w})\right)$. More about twisted convolution can be found in [1-3].

In [4], the authors defined the Hardy space $H_{L}^{1}\left(\mathbb{C}^{n}\right)$ associated with a twisted convolution. They gave several characterizations of $H_{L}^{1}\left(\mathbb{C}^{n}\right)$ via maximal functions, the atomic decomposition, and the behavior of the local Riesz transform. As applications, the boundedness of Hömander multipliers on Hardy spaces is considered in [5]. The 'twisted cancelation' and Weyl multipliers were introduced for the first time in [6]. Recently, Huang and Wang [7] defined the Hardy space $H_{L}^{p}\left(\mathbb{C}^{n}\right)$ associated with a twisted convolution for $\frac{2 n}{2 n+1}<p \leq 1$. Huang gave the characterizations of the Hardy space associated with twisted convolution by the Lusin area integral function and the Littlewood-Paley function defined by the heat kernel in [8] and established the boundedness of the Weyl multiplier by these characterizations in [9]. Recently, Huang and Liu gave the molecular characterization of Hardy space associated with twisted convolution in [10]. The purpose of this paper is to give some new real-variable characterizations for $H_{L}^{p}\left(\mathbb{C}^{n}\right)$, including the Poisson maximal function, the Lusin area integral, and the Littlewood-Paley $g$-function defined by the Poisson kernel.

We first give some basic notations concerning $H_{L}^{p}\left(\mathbb{C}^{n}\right)$. Let $\mathcal{B}$ denote the class of $C^{\infty}$ functions $\varphi$ on $\mathbb{C}^{n}$, supported on the ball $B(0,1)$ such that $\|\varphi\|_{\infty} \leq 1$ and $\|\nabla \varphi\|_{\infty} \leq 2$. For $t>0$, let $\varphi_{t}(z)=t^{-2 n} \varphi(z / t)$. Given $\sigma>0,0<\sigma \leq+\infty$, and a tempered distribution $f$, define the grand maximal function

$$
M_{\sigma} f(z)=\sup _{\varphi \in \mathcal{B}} \sup _{0<t<\sigma}\left|\varphi_{t} \times f(z)\right|
$$

Then the Hardy space $H_{L}^{p}\left(\mathbb{C}^{n}\right)$ can be defined by

$$
H_{L}^{p}\left(\mathbb{C}^{n}\right)=\left\{f \in \mathcal{S}^{\prime}\left(\mathbb{C}^{n}\right): M_{\infty} f \in L^{p}\left(\mathbb{C}^{n}\right)\right\}
$$

For any $f \in H_{L}^{p}\left(\mathbb{C}^{n}\right)$, define $\|f\|_{H_{L}^{p}\left(\mathbb{C}^{n}\right)}=\left\|M_{\infty} f\right\|_{L^{p}}$.

Definition 1 Let $\frac{2 n}{2 n+1}<p \leq 1 \leq q \leq \infty$ and $p \neq q$. A function $a(z)$ is a $H_{L}^{p, q}$-atom for the Hardy space $H_{L}^{p}\left(\mathbb{C}^{n}\right)$ associated to a ball $B\left(z_{0}, r\right)$ if

$$
\begin{aligned}
& \text { (1) } \operatorname{supp} a \subset B\left(z_{0}, r\right) ; \\
& \text { (2) }\|a\|_{q} \leq\left|B\left(z_{0}, r\right)\right|^{1 / q-1 / p} ; \\
& \text { (3) } \int_{\mathbb{C}^{n}} a(w) \bar{\omega}\left(z_{0}, w\right) d w=0 .
\end{aligned}
$$

We define the atomic Hardy space $H_{L}^{p, q}\left(\mathbb{C}^{n}\right)$ to be the set of all tempered distributions of the form $\sum_{j} \lambda_{j} a_{j}$ (the sum converges in the topology of $\mathcal{S}^{\prime}\left(\mathbb{C}^{n}\right)$ ), where $a_{j}$ are $H_{L}^{p, q}$-atoms and $\sum_{j}\left|\lambda_{j}\right|^{p}<+\infty$. 
The atomic quasi-norm in $H_{L}^{p, q}\left(\mathbb{C}^{n}\right)$ is defined by

$$
\|f\|_{L \text {-atom }}=\inf \left\{\left(\sum_{j}\left|\lambda_{j}\right|^{p}\right)^{1 / p}\right\},
$$

where the infimum is taken over all decompositions $f=\sum_{j} \lambda_{j} a_{j}$ and $a_{j}$ are $H_{L}^{p, q}$-atoms.

The following result has been proved in [4] and [7].

Proposition 1 Let $\frac{2 n}{2 n+1}<p \leq 1$. Then for a tempered distribution $f$ on $\mathbb{C}^{n}$, the following are equivalent:

(i) $M_{\infty} f \in L^{p}\left(\mathbb{C}^{n}\right)$.

(ii) For some $\sigma, 0<\sigma<+\infty, M_{\sigma} f \in L^{p}\left(\mathbb{C}^{n}\right)$.

(iii) For some radial function $\varphi \in \mathcal{S}$, such that $\int_{\mathbb{C}^{n}} \varphi(z) d z \neq 0$, we have

$$
\sup _{0<t<1}\left|\varphi_{t} \times f(z)\right| \in L^{p}\left(\mathbb{C}^{n}\right)
$$

(iv) $f$ can be decomposed as $f=\sum_{j} \lambda_{j} a_{j}$, where $a_{j}$ are $H_{L}^{p, q}$-atoms and $\sum_{j}\left|\lambda_{j}\right|^{p}<+\infty$.

Corollary 1 Let $\frac{2 n}{2 n+1}<p \leq 1$ and $1<q \leq \infty$. Then $H_{L}^{p, q}\left(\mathbb{C}^{n}\right)=H_{L}^{p}\left(\mathbb{C}^{n}\right)$ with equivalent norms.

Let $\left\{P_{t}^{L}\right\}_{t>0}$ be the Poisson semigroup generated by the operator $L$. Then, for $f \in L^{2}\left(\mathbb{C}^{n}\right)$, the function $e^{-t \sqrt{L}} f$ has the special Hermite expansion ( $c f$. [11])

$$
e^{-t \sqrt{L}} f(z)=(2 \pi)^{-n} \sum_{k=0}^{\infty} e^{-\sqrt{2 k+n} t} f \times \varphi_{k}(z)
$$

where $\varphi_{k}$ are Laguerre functions. Therefore $e^{-t \sqrt{L}} f$ is given by the twisted convolution with the kernel

$$
P_{t}(z)=(2 \pi)^{-n} \sum_{k=0}^{\infty} e^{-\sqrt{2 k+n} t} \varphi_{k}(z)
$$

The Poisson maximal function is defined by

$$
M_{P}(f)(z)=\sup _{t>0}\left|P_{t} \times f(z)\right|
$$

We can characterize the Hardy space $H_{L}^{1}\left(\mathbb{C}^{n}\right)$ as follows.

Theorem $1 f \in H_{L}^{1}\left(\mathbb{C}^{n}\right)$ if and only if $f \in L^{1}\left(\mathbb{C}^{n}\right)$ and $M_{P}(f) \in L^{1}\left(\mathbb{C}^{n}\right)$. Moreover, we have

$$
\|f\|_{H_{L}^{1}} \sim\left\|M_{P}(f)\right\|_{L^{1}} .
$$

We define the area integral associated to $\left\{P_{t}^{L}\right\}_{t>0}$ by

$$
\left(S_{L}^{k} f\right)(z)=\left(\int_{0}^{+\infty} \int_{|z-w|<t}\left|D_{t}^{k} f(w)\right|^{2} \frac{d w d t}{t^{2 n+1}}\right)^{1 / 2},
$$


the Littlewood-Paley $g$-function by

$$
\mathcal{G}_{L}^{k}(f)(z)=\left(\int_{0}^{\infty}\left|D_{t}^{k} f(z)\right|^{2} \frac{d t}{t}\right)^{1 / 2}
$$

and we consider the $g_{\lambda}^{*}$-function associated with $L$ defined by

$$
g_{\lambda, k}^{*} f(z)=\left(\int_{0}^{\infty} \int_{\mathbb{C}^{n}}\left(\frac{t}{t+|z-w|}\right)^{2 \lambda n}\left|D_{t}^{k} f(w)\right|^{2} \frac{d w d t}{t^{2 n+1}}\right)^{1 / 2}
$$

where $D_{t}^{k} f(z)=t^{k}\left(\partial_{t}^{k} P_{t}^{L} f\right)(z)$.

Now we can prove the main result of this paper.

\section{Theorem 2}

(a) A function $f \in H_{L}^{1}\left(\mathbb{C}^{n}\right)$ if and only if its Lusin area integral $S_{L}^{k} f \in L^{1}\left(\mathbb{C}^{n}\right)$ and $f \in L^{1}\left(\mathbb{C}^{n}\right)$. Moreover, we have

$$
\|f\|_{H_{L}^{1}} \sim\left\|S_{L}^{k} f\right\|_{L^{1}} .
$$

(b) A function $f \in H_{L}^{1}\left(\mathbb{C}^{n}\right)$ if and only if its Littlewood-Paley g-function $\mathcal{G}_{L}^{k} f \in L^{1}\left(\mathbb{C}^{n}\right)$ and $f \in L^{1}\left(\mathbb{C}^{n}\right)$. Moreover, we have

$$
\|f\|_{H_{L}^{1}} \sim\left\|\mathcal{G}_{L}^{k} f\right\|_{L^{1}}
$$

(c) A function $f \in H_{L}^{1}\left(\mathbb{C}^{n}\right)$ if and only if its $g_{\lambda}^{*}$-function $g_{\lambda, k}^{*} f \in L^{1}\left(\mathbb{C}^{n}\right)$ and $f \in L^{1}\left(\mathbb{C}^{n}\right)$, where $\lambda>3$. Moreover, we have

$$
\|f\|_{H_{L}^{1}} \sim\left\|g_{\lambda, k}^{*} f\right\|_{L^{1}}
$$

Remark 1 In this paper, we just give the proofs of our results for $p=1$. In fact, we can prove the case $\frac{2 n}{2 n+1}<p<1$ under more conditions (such as that $f$ vanishes weakly at infinity). The proofs of the case $\frac{2 n}{2 n+1}<p<1$ are quite similar to the case $p=1$, so we omit them.

Throughout the article, we will use $C$ to denote a positive constant, which is independent of the main parameters and may be different at each occurrence. By $B_{1} \sim B_{2}$, we mean that there exists a constant $C>1$ such that $\frac{1}{C} \leq \frac{B_{1}}{B_{2}} \leq C$.

\section{Preliminaries}

In this section, we give some preliminaries that we will use in the sequel.

Let $K_{t}(z)$ be the heat kernel of $\left\{T_{t}^{L}\right\}_{t>0}$. Then we can get (cf. [11])

$$
K_{t}(z)=(4 \pi)^{-n}(\sinh t)^{-n} e^{-\frac{1}{4}|z|^{2}(\operatorname{coth} t)} .
$$

It is easy to prove that the heat kernel $K_{t}(z)$ has the following estimates ( $c f$. [8]).

Lemma 1 There exists a positive constant $C>0$ such that

(i) $\left|K_{t}(z)\right| \leq C t^{-n} e^{-C \frac{|z|^{2}}{t}}$;

(ii) $\left|\nabla K_{t}(z)\right| \leq C t^{-n-\frac{1}{2}} e^{-C \frac{|z|^{2}}{t}}$. 
Let $Q_{t}^{k}(z)$ be the twisted convolution kernel of $Q_{t}^{k}=\left.t^{2 k} \partial_{s}^{k} T_{s}^{L}\right|_{s=t^{2}}$. Then

$$
Q_{t}^{k}(z)=\left.t^{2 k} \partial_{s}^{k} K_{s}(z)\right|_{s=t^{2}}
$$

We have the following estimates [8].

Lemma 2 There exist constants $C, C_{k}>0$ such that

(i) $\left|Q_{t}^{k}(z)\right| \leq C_{k} t^{-2 n} e^{-C t^{-2}|z|^{2}}$;

(ii) $\left|\nabla Q_{t}^{k}(z)\right| \leq C_{k} t^{-2 n-1} e^{-C t^{-2}|z|^{2}}$.

By the subordination formula, we can give the following estimates as regards the Poisson kernel.

Lemma 3 There exist constants $C_{k}>0, A>0$ such that

(a)

$$
0<P_{t}(z) \leq C_{k} \frac{t}{\left(t^{2}+A|z|^{2}\right)^{(2 n+1) / 2}}
$$

(b)

$$
\left|\nabla P_{t}(z)\right| \leq C_{k} \frac{\sqrt{t}}{\left(t^{2}+A|z|^{2}\right)^{(2 n+1) / 2}}
$$

Lemma 4 Let $D_{t}^{k}(z)$ be the integral kernel of the operator $D_{t}^{k}$. Then there exist constants $C_{k}>0, A>0$, such that

(a)

$$
\left|D_{t}^{k}(z)\right| \leq C_{k} \frac{t}{\left(t^{2}+A|z|^{2}\right)^{(2 n+1) / 2}}
$$

(b)

$$
\left|\nabla D_{t}^{k}(z)\right| \leq C_{k} \frac{\sqrt{t}}{\left(t^{2}+A|z|^{2}\right)^{(2 n+1) / 2}}
$$

We also need some basic properties about the tent space ( $c f .[12])$.

Let $0<p<\infty$, and $1 \leq q \leq \infty$. Then the tent space $T_{q}^{p}$ is defined as the space of functions $f$ on $\mathbb{C}^{n} \times \mathbb{R}^{+}$, so that

$$
\left(\int_{\Gamma(z)}|f(w, t)|^{q} \frac{d w d t}{t^{2 n+1}}\right)^{1 / q} \in L^{p}\left(\mathbb{C}^{n}\right), \quad \text { when } 1 \leq q<\infty
$$

and

$$
\sup _{(w, t) \in \Gamma(z)}|f(w, t)| \in L^{p}\left(\mathbb{C}^{n}\right), \quad \text { when } q=\infty
$$

where $\Gamma(z)$ is the standard cone whose vertex is $z \in \mathbb{C}^{n}$, i.e.,

$$
\Gamma(z)=\{(w, t):|w-z|<t\} .
$$


Assume $B\left(z_{0}, r\right)$ is a ball in $\mathbb{C}^{n}$, its tent $\hat{B}$ is defined by $\hat{B}=\left\{(w, t):\left|w-z_{0}\right| \leq r-t\right\}$. A function $a(z, t)$ supported in a tent $\hat{B}, B$ a ball in $\mathbb{C}^{n}$, is said to be an atom in the tent space $T_{q}^{p}$ if and only if it satisfies

$$
\left(\int_{\hat{B}}|a(z, t)|^{2} \frac{d z d t}{t}\right)^{1 / 2} \leq|B|^{1 / 2-1 / p}
$$

The atomic decomposition of $T_{q}^{p}$ is stated as follows.

Proposition 2 When $0<p \leq 1$, then for any $f \in T_{2}^{p}$ can be written as $f=\sum \lambda_{k} a_{k}$, where $a_{k}$ are atoms and $\sum\left|\lambda_{k}\right|^{p} \leq C\|f\|_{T_{2}^{p}}^{p}$.

\section{The proofs of the main results}

Let

$$
M_{H} f(z)=\sup _{t>0}\left|K_{t} \times f(z)\right|, \quad f \in L^{1}\left(\mathbb{C}^{n}\right)
$$

be the heat maximal function. Then we can characterize $H_{L}^{1}\left(\mathbb{C}^{n}\right)$ by the maximal function $M_{H} f$ as follows (cf. [4] or [8]).

Lemma $5 f \in H_{L}^{1}\left(\mathbb{C}^{n}\right)$ if and only if $M_{H} f \in L^{1}\left(\mathbb{C}^{n}\right)$ and $f \in L^{1}\left(\mathbb{C}^{n}\right)$.

Now, we give the proof of Theorem 1.

Proof of Theorem 1 If $f \in H_{L}^{1}\left(\mathbb{C}^{n}\right)$, then, by Lemma 5 , we get $M_{H} f \in L^{1}\left(\mathbb{C}^{n}\right)$. Since

$$
P_{t}(z)=\frac{1}{\sqrt{\pi}} \int_{0}^{\infty} K_{t^{2} / 4 \mu}(z) e^{-\mu} \mu^{-1 / 2} d \mu
$$

we have $\left\|M_{P}(f)\right\|_{L^{1}} \leq C\left\|M_{H}(f)\right\|_{L^{1}}$, i.e., $M_{P} f \in L^{1}\left(\mathbb{C}^{n}\right)$.

For the reverse, there exists a function $\eta$ defined on $(1, \infty)$ that is rapidly decreasing at $\infty$ and satisfies the moment conditions (cf. [13])

$$
\int_{1}^{\infty} \eta(t) d t=1, \quad \int_{1}^{\infty} t^{k} \eta(t) d t=0, \quad k=1,2, \ldots
$$

Let

$$
\Phi(z)=\int_{1}^{\infty} \eta(t) P_{t}(z) d t .
$$

Since

$$
\left(1+s^{2}\right)^{-(2 n+1) / 2}=\sum_{k<R} a_{k} s^{k}+O\left(s^{R}\right), \quad 0 \leq s<\infty
$$

for appropriate binomial coefficients $a_{k}$, we have

$$
\frac{t}{\left(t^{2}+A|z|^{2}\right)^{(2 n+1) / 2}}=\sum_{k<R} a_{k} t|z|^{-1-2 n}\left(\frac{t}{|z|}\right)^{k}+O\left(t^{R+1}|z|^{-2 n-1-R}\right) .
$$


By (8) and Lemma 3, we know that $\Phi$ and any derivative of $\Phi$ are rapidly decreasing. Thus $\Phi \in \mathcal{S}$ and

$$
\int_{\mathbb{C}^{n}} \Phi(z) d z=\int_{1}^{\infty} \eta(t) d t=1
$$

Therefore,

$$
M_{\Phi}(f)(z) \leq M_{P}(f)(z) \int_{1}^{\infty}|\eta(t)| d t \leq C M_{P}(f)(z) .
$$

This proves that $M_{P}(f) \in L^{1}\left(\mathbb{C}^{n}\right)$ implies $f \in H_{L}^{1}\left(\mathbb{C}^{n}\right)$ and the proof of Theorem 1 is complete.

In order to get our results, we need the following lemma ( $c f$. Lemma 5 in [8]).

\section{Lemma 6}

(i) The operators $S_{L}^{k}$ and $\mathcal{G}_{L}^{k}$ are isometries on $L^{2}\left(\mathbb{C}^{n}\right)$ up to constant factors. Exactly,

$$
\left\|\mathcal{G}_{L}^{k} f\right\|_{L^{2}} \sim\|f\|_{L^{2}}, \quad\left\|S_{L}^{k} f\right\|_{L^{2}} \sim\|f\|_{L^{2}}
$$

(ii) When $\lambda>1$, there exists a constant $C>0$, such that

$$
C^{-1}\|f\|_{L^{2}} \leq\left\|g_{\lambda, k}^{*} f\right\|_{L^{2}} \leq C\|f\|_{L^{2}}
$$

We define the new Lusin type area integral operator by

$$
\left(S_{L, \alpha}^{k} f\right)(z)=\left(\int_{0}^{+\infty} \int_{|z-w|<\alpha t}\left|D_{t}^{k} f(w)\right|^{2} \frac{d w d t}{t^{2 n+1}}\right)^{1 / 2}
$$

where $\alpha>0$.

Lemma 7 It is easy to see that the above definition of the area integral operator is independent of $\alpha$ in the sense of $\left\|\left(S_{L}^{\alpha} f\right)\right\|_{L^{p}} \sim\left\|\left(S_{L}^{\beta} f\right)\right\|_{L^{p}}$, for $0<\alpha<\beta<\infty$ and $0<p<\infty$ (cf. [12]). In the following, we use $S_{L}^{k}$ to denote $S_{L, 1}^{k}$.

Proof of Theorem 2 (a) By Lemma 4, we can prove that there exists a constant $C>0$ such that for any atom $a(z)$ of $H_{L}^{1}\left(\mathbb{C}^{n}\right)$, we have

$$
\left\|S_{L}^{k} a\right\|_{L^{1}} \leq C
$$

In the following, we will show that $f \in H_{L}^{1}\left(\mathbb{C}^{n}\right)$ when $S_{L}^{k} f \in L^{1}\left(\mathbb{C}^{n}\right)$ and $f \in L^{1}\left(\mathbb{C}^{n}\right)$.

We first assume that $f \in L^{1}\left(\mathbb{C}^{n}\right) \cap L^{2}\left(\mathbb{C}^{n}\right)$. When $S_{L}^{k} f \in L^{1}\left(\mathbb{C}^{n}\right)$, we know $D_{t}^{k} f \in T_{2}^{1}$. By Proposition 2, we get

$$
D_{t}^{k} f(z)=\sum_{j} \lambda_{j} a_{j}(z, t)
$$


where $a_{j}(z, t)$ are atoms of $T_{2}^{1}$ and $\sum_{j}\left|\lambda_{j}\right|<\infty$. By the spectrum theorem (cf. [14]), we can prove

$$
f(z)=4 \int_{0}^{\infty} D_{t}^{k}\left(D_{t}^{k} f(z)\right) \frac{d t}{t}
$$

By (10) and (11), we get

$$
f(z)=4 \int_{0}^{+\infty} D_{t}^{k}\left(\sum_{j} \lambda_{j} a_{j}(z, t)\right) \frac{d t}{t}=C \sum_{j} \lambda_{j} \int_{0}^{+\infty} D_{t}^{k} a_{j}(z, t) \frac{d t}{t} .
$$

Therefore, it is sufficient to prove $\alpha_{j}=\int_{0}^{+\infty} D_{t}^{k} a_{j}(z, t) \frac{d t}{t}, i=1,2, \ldots$, are bounded in $H_{L}^{1}\left(\mathbb{C}^{n}\right)$ uniformly, i.e., there exists a constant $C>0$ such that for any atom $a(z, t)$ in $T_{2}^{1}$,

$$
\|\alpha\|_{H_{L}^{1}}=\left\|\int_{0}^{+\infty} D_{t}^{k} a(z, t) \frac{d t}{t}\right\|_{H_{L}^{1}} \leq C .
$$

We assume that $a(z, t)$ is supported in $\hat{B}\left(z_{0}, r\right)$, where $\hat{B}\left(z_{0}, r\right)$ denotes the tent of the ball $B\left(z_{0}, r\right)$, then

$$
\left\|\sup _{t>0}\left|e^{-t \sqrt{L}} \alpha(z)\right|\right\|_{L^{1}} \leq\left\|\left(\sup _{t>0}\left|e^{-t \sqrt{L}} \alpha(z)\right|\right) \chi_{B^{*}}\right\|_{L^{1}}+\left\|\left(\sup _{t>0}\left|e^{-t \sqrt{L}} \alpha(z)\right|\right) \chi_{\left(B^{*}\right)^{c}}\right\|_{L^{1}}=I_{1}+I_{2},
$$

where $B^{*}=B\left(z_{0}, 2 r\right)$.

By the Hölder inequality, we get

$$
I_{1} \leq\left|B^{*}\right|^{1 / 2}\left(\int_{\mathbb{C}^{n}}\left(\sup _{t>0}\left|e^{-t \sqrt{L}} \alpha(z)\right|\right)^{2} d z\right)^{1 / 2} \leq\left|B^{*}\right|^{1 / 2}\|\alpha\|_{L^{2}} .
$$

By the self-adjointness of $D_{t}^{k}$ and Lemma 5, we can get

$$
\begin{aligned}
\|\alpha\|_{L^{2}}= & \sup _{\|\beta\|_{L^{2}} \leq 1} \int_{\mathbb{C}^{n}} \alpha(z) \bar{\beta}(z) d z \\
= & \sup _{\|\beta\|_{L^{2}} \leq 1} \int_{\mathbb{C}^{n}}\left(\int_{0}^{+\infty} D_{t}^{k} a(z, t) \frac{d t}{t}\right) \bar{\beta}(z) d z \\
= & \sup _{\|\beta\|_{L^{2}} \leq 1} \int_{0}^{+\infty} \int_{\mathbb{C}^{n}} D_{t}^{k} a(z, t) \bar{\beta}(z) d z \frac{d t}{t} \\
= & \sup _{\|\beta\|_{L^{2}} \leq 1} \int_{0}^{+\infty} \int_{\mathbb{C}^{n}} a(z, t) D_{t}^{k} \bar{\beta}(z) d z \frac{d t}{t} \\
\leq & \sup _{\|\beta\|_{L^{2}} \leq 1}\left(\int_{\mathbb{C}^{n}} \int_{0}^{+\infty}|a(z, t)|^{2} \frac{d z d t}{t}\right)^{1 / 2} \\
& \times\left(\int_{\mathbb{C}^{n}} \int_{0}^{+\infty}\left|D_{t}^{k} \bar{\beta}(z)\right|^{2} \frac{d z d t}{t}\right)^{1 / 2} \\
\leq & |B|^{-1 / 2}\|\beta\|_{L^{2}} \leq|B|^{-1 / 2} .
\end{aligned}
$$

This gives the proof of $I_{1} \leq C$. 
By Lemma 2, we can prove

$$
\begin{aligned}
& \sup _{s>0}\left|e^{-s \sqrt{L}} \int_{0}^{+\infty} D_{t}^{k} a(z, t) \frac{d t}{t}\right| \\
&=\sup _{s>0}\left|e^{-s \sqrt{L}} \int_{0}^{+\infty}(-t \sqrt{L})^{k} e^{-t \sqrt{L}} a(z, t) \frac{d t}{t}\right| \\
&=\sup _{s>0}\left|\int_{0}^{+\infty}(-t \sqrt{L})^{k} e^{-(s+t) \sqrt{L}} a(z, t) \frac{d t}{t}\right| \\
&=\sup _{s>0}\left|\int_{0}^{+\infty}\left(\frac{t}{s+t}\right)^{k}(-(s+t) \sqrt{L})^{k} e^{-(s+t) \sqrt{L}} a(z, t) \frac{d t}{t}\right| \\
&=\sup _{s>0}\left|\int_{0}^{+\infty}\left(\frac{t}{s+t}\right)^{k} \int_{\mathbb{C}^{n}} D_{s+t}^{k}(z-w) a(w, t) \frac{d w d t}{t}\right| \\
& \leq \sup _{s>0} \int_{0}^{+\infty} \frac{t}{s+t} \int_{\mathbb{C}^{n}} \frac{s+t}{\left((s+t)^{2}+A|z-w|^{2}\right)^{(2 n+1) / 2}}|a(w, t)| \frac{d w d t}{t} \\
& \leq \sup _{s>0}\left(\int_{0}^{r} \int_{B}(s+t)^{-4 n}\left(1+A \frac{|z-w|^{2}}{(s+t)^{2}}\right)^{-(2 n+1)}\left(\frac{t}{s+t}\right)^{2} \frac{d w d t}{t}\right)^{1 / 2} \\
& \leq \times\left(\int_{0}^{r} \int_{B}|a(w, t)|^{2} \frac{d w d t}{t}\right)^{1 / 2} \\
& \leq|B|^{-1 / 2}\left|z-z_{0}\right|^{-(2 n+1)}\left(\int_{0}^{r} \int_{B} t d w d t\right)^{1 / 2} \\
& \quad C r\left|z-z_{0}\right|^{-(2 n+1)} .
\end{aligned}
$$

Then we get

$$
I_{2} \leq C r \int_{\left(B^{*}\right)^{c}}\left|z-z_{0}\right|^{-(2 n+1)} d z \leq C
$$

When $f \in L^{1}\left(\mathbb{C}^{n}\right)$, we can proceed similarly to Proposition 14 in [15]. In fact, we let $f_{s}=$ $T_{2^{-s}}^{L} f, s \geq 0$. Then, by $f \in L^{1}\left(\mathbb{C}^{n}\right)$ and Lemma 3, we know $f_{s} \in L^{2}\left(\mathbb{C}^{n}\right)$ and $\left\|S_{L}^{k} f_{s}\right\|_{1} \leq\left\|S_{I}^{k} f\right\|_{1}$. By the above proof, we get

$$
\left\|f_{s}\right\|_{H_{L}^{1}\left(\mathbb{C}^{n}\right)} \lesssim\left\|S_{L}^{k} f_{s}\right\|_{L^{1}} \leq\left\|S_{L}^{k} f\right\|_{L^{1}}
$$

By the monotone convergence theorem, we have

$$
\left\|f_{s}-f_{n}\right\|_{H_{L}^{1}} \leq\left\|S_{L}^{k}\left(f_{s}-f_{n}\right)\right\|_{L^{1}} \rightarrow 0, \quad \text { when } s, n \rightarrow+\infty
$$

Therefore, $\left\{f_{s}\right\}$ is a Cauchy sequence in $H_{L}^{1}\left(\mathbb{C}^{n}\right)$ and there exists $g \in H_{L}^{1}\left(\mathbb{C}^{n}\right)$ such that

$$
\lim _{s \rightarrow+\infty} f_{s}=g \quad \text { in } H_{L}^{1}\left(\mathbb{C}^{n}\right)
$$

As

$$
\lim _{s \rightarrow+\infty} f_{s}=f \quad \text { in }\left(B M O_{L}\right)^{*}
$$

we know $f=g \in H_{L}^{1}\left(\mathbb{C}^{n}\right)$ and $\|f\|_{H_{L}^{1}\left(\mathbb{C}^{n}\right)} \lesssim\left\|S_{L}^{k} f\right\|_{L^{1}}$. 
This gives the proof of Theorem 2(a).

(b) Firstly, by Lemma 4, we can prove that there exists a positive constant $C$ such that for any atom $a(z)$ of $H_{L}^{1}\left(\mathbb{C}^{n}\right)$, we have

$$
\left\|\mathcal{G}_{L}^{k} a\right\|_{L^{1}} \leq C
$$

For the reverse, by (a), it is sufficient to prove

$$
\left\|S_{L}^{k+1} f\right\|_{L^{1}} \leq C\left\|\mathcal{G}_{L}^{k} f\right\|_{L^{1}}
$$

Our proof is motivated by [16]. Let

$$
F(z)(t)=\left(\partial_{t}^{k} e^{-t \sqrt{L}} f\right)(z), \quad V(z, s)=e^{-s \sqrt{L}} F(z) .
$$

Then

$$
V(z, s)(t)=e^{-s \sqrt{L}}\left(\partial_{t}^{k} e^{-t \sqrt{L}} f\right)(z)=\left(\partial_{t}^{k} e^{-(s+t) \sqrt{L}} f\right)(z) .
$$

Therefore

$$
\begin{aligned}
\int_{0}^{+\infty}|V(z, s)(t)|^{2} t^{2 k-1} d t & =\int_{0}^{+\infty}\left|\left(\partial_{t}^{k} e^{-(s+t) \sqrt{L}} f\right)(z)\right|^{2} t^{2 k-1} d t \\
& =\int_{s}^{+\infty}\left|\left(\partial_{t}^{k} e^{-t \sqrt{L}} f\right)(z)\right|^{2}(t-s)^{2 k-1} d t .
\end{aligned}
$$

Hence

$$
\sup _{s>0} \int_{0}^{+\infty}|V(z, s)(t)|^{2} t^{2 k-1} d t \leq \int_{0}^{+\infty}\left|\left(t^{k} \partial_{t}^{k} e^{-t \sqrt{L}} f\right)(z)\right|^{2} \frac{d t}{t}=\left(\mathcal{G}_{L}^{k} f(z)\right)^{2} .
$$

Let $\mathbf{X}=L^{2}\left((0, \infty), t^{2 k-1} d t\right)$. Then

$$
\sup _{s>0}\left\|e^{-s \sqrt{L}} F(z)\right\|_{\mathbf{X}}=\mathcal{G}_{L}^{k} f(z) \in L^{1}\left(\mathbb{C}^{n}\right) .
$$

Therefore $F \in H_{\mathbf{X}}^{1}\left(\mathbb{C}^{n}\right)$, here $H_{\mathbf{X}}^{1}\left(\mathbb{C}^{n}\right)$ can be seen as a vector-valued Hardy space. This shows that $\widetilde{S_{L}^{1}} F(z) \in L^{1}\left(\mathbb{C}^{n}\right)$, where

$$
\widetilde{S}_{L}^{1} F(z)=\left(\int_{0}^{+\infty} \int_{|z-w|<2 t}\left\|D_{t}^{1} F(w)\right\|_{\mathbf{X}}^{2} \frac{d w d t}{t^{2 n+1}}\right)^{1 / 2} .
$$

By

$$
\begin{aligned}
\left(S_{L}^{1} F(z)\right)^{2} & =\int_{0}^{+\infty} \int_{|z-w|<2 t}\left\|D_{t}^{1}(z)\right\|_{\mathbf{X}}^{2} \frac{d w d t}{t^{2 n+1}} \\
& =\int_{0}^{+\infty} \int_{|z-w|<2 t} \int_{0}^{+\infty}\left|(-t \sqrt{L}) e^{-t \sqrt{L}} F(w)(s)\right|^{2} s^{2 k-1} d s \frac{d w d t}{t^{2 n+1}} \\
& =\int_{0}^{+\infty} \int_{0}^{+\infty} \int_{|z-w|<2 t}\left|(-\sqrt{L})^{k+1} e^{-(s+t) \sqrt{L}} f(w)\right|^{2} t^{1-2 n} s^{2 k-1} d w d t d s
\end{aligned}
$$




$$
\begin{aligned}
& =\int_{0}^{+\infty} \int_{s}^{+\infty} \int_{|z-w|<2(t-s)}\left|(-\sqrt{L})^{k+1} e^{-t \sqrt{L}} f(w)\right|^{2}(t-s)^{1-2 n} s^{2 k-1} d w d t d s \\
& =\int_{0}^{+\infty} \int_{0}^{t} \int_{|z-w|<2(t-s)}\left|(-\sqrt{L})^{k+1} e^{-t \sqrt{L}} f(w)\right|^{2}(t-s)^{1-2 n} s^{2 k-1} d w d s d t \\
& \geq \int_{0}^{+\infty} \int_{0}^{t / 2} \int_{|z-w|<2(t-s)}\left|(-\sqrt{L})^{k+1} e^{-t \sqrt{L}} f(w)\right|^{2}(t-s)^{1-2 n} s^{2 k-1} d w d s d t \\
& \geq \int_{0}^{+\infty} \int_{0}^{t / 2} \int_{|z-w|<t}\left|(-\sqrt{L})^{k+1} e^{-t \sqrt{L}} f(w)\right|^{2} t^{1-2 n} s^{2 k-1} d w d s d t \\
& =\frac{1}{2 k 2^{2 k}} \int_{0}^{+\infty} \int_{|z-w|<t}\left|(-t \sqrt{L})^{k+1} e^{-t \sqrt{L}} f(w)\right|^{2} t^{-1-2 n} d w d t \\
& =\frac{1}{2 k 2^{2 k}} \int_{0}^{+\infty} \int_{|z-w|<t}\left|D_{t}^{k+1} f(w)\right|^{2} \frac{d w d t}{t^{2 n+1}}=\frac{1}{2 k 2^{2 k}}\left(S_{L}^{k+1} f(z)\right)^{2},
\end{aligned}
$$

we get $S_{L}^{k+1} f \in L^{1}\left(\mathbb{C}^{n}\right)$. Then $f \in H_{L}^{1}\left(\mathbb{C}^{n}\right)$ follows from (a).

This completes the proof of Theorem 2(b).

(c) By $S_{L}^{k} f(z) \leq\left(\frac{1}{2}\right)^{2 \lambda n} g_{\lambda, k}^{*} f(z)$, we know $f \in H_{L}^{1}\left(\mathbb{C}^{n}\right)$ when $g_{\lambda, k}^{*} f \in L^{1}\left(\mathbb{C}^{n}\right)$ and $f \in L^{1}\left(\mathbb{C}^{n}\right)$. In the following, we show there exists a constant $C>0$ such that for any atom $a(z)$ of $H_{L}^{1}\left(\mathbb{C}^{n}\right)$, we have

$$
\left\|g_{\lambda, k}^{*} a\right\|_{L^{1}} \leq C
$$

Without loss of generality, we may assume $a(z)$ is supported in $B(0, r)$, then

$$
\begin{aligned}
g_{\lambda, k}^{*} a(z)^{2}= & \int_{0}^{\infty} \int_{\mathbb{C}^{n}}\left(\frac{t}{t+|z-w|}\right)^{2 \lambda n}\left|D_{t}^{k} a(w)\right|^{2} \frac{d w d t}{t^{2 n+1}} \\
= & \int_{0}^{\infty} \int_{|z-w|<t}\left(\frac{t}{t+|z-w|}\right)^{2 \lambda n}\left|D_{t}^{k} a(w)\right|^{2} \frac{d w d t}{t^{2 n+1}} \\
& +\sum_{i=1}^{\infty} \int_{0}^{\infty} \int_{2^{i-1} t \leq|z-w|<2^{i} t}\left(\frac{t}{t+|z-w|}\right)^{2 \lambda n}\left|D_{t}^{k} a(w)\right|^{2} \frac{d w d t}{t^{2 n+1}} \\
\leq & C S_{L}^{1} a(z)^{2}+\sum_{i=1}^{\infty} 2^{-2 i \lambda n} S_{L, 2^{i}}^{k} a(z)^{2} .
\end{aligned}
$$

Therefore,

$$
\left\|g_{\lambda, k}^{*} a\right\|_{L^{1}} \leq C\left\|S_{L}^{1} a\right\|_{L^{1}}+\sum_{i=1}^{\infty} 2^{-i \lambda n}\left\|S_{L, i^{i}}^{k} a\right\|_{L^{1}} .
$$

By part (a), we have $\left\|S_{L}^{k} a\right\|_{L^{1}} \leq C$. In the following, we will prove that

$$
\left\|S_{L, 2^{i}}^{k} a\right\|_{L^{1}} \leq C 2^{3 i n} .
$$

First, by Lemma 5, we can obtain

$$
\left\|S_{L, 2^{i}}^{k} a\right\|_{L^{1}\left(B\left(0,2^{i+2} r\right)\right)} \leq\left|B\left(0,2^{i+2} r\right)\right|^{1 / 2}\left\|S_{L, 2^{i}}^{k} a\right\|_{L^{2}} \leq C 2^{2 i n} .
$$


Let $z \notin B\left(0,2^{i+2} r\right)$. We have

$$
\begin{aligned}
S_{L, 2^{i}}^{k} a(z)^{2} & \leq \int_{0}^{\infty} \int_{|z-w|<2^{i} t}\left(\int_{B(0, r)}\left|D_{t}^{k}(w-v)-D_{t}^{k}(w)\right||a(v)| d v\right)^{2} \frac{d w d t}{t^{2 n+1}} \\
& \leq \int_{0}^{\frac{|z|}{2^{i+1}}} \int_{|z-w|<2^{i} t}(\cdots)^{2} \frac{d w d t}{t^{2 n+1}}+\int_{\frac{|z|}{2^{i+1}}}^{\infty} \int_{|z-w|<2^{i} t}(\cdots)^{2} \frac{d w d t}{t^{2 n+1}} \\
& =I_{1}+I_{2} .
\end{aligned}
$$

For $z \notin B\left(0,2^{i+2} r\right)$, when $|z-w|<2^{i} t \leq \frac{|z|}{2}$, we have $|w| \sim|z|$. By Lemma 4 , we get

$$
\begin{aligned}
I_{1} & \leq C \int_{0}^{\frac{|z|}{2^{i+1}}} \int_{|z-w|<2^{i} t}\left(\int_{B(0, r)} \frac{\sqrt{t}}{\left(t^{2}+A|w|^{2}\right)^{(2 n+1) / 2}}|v||a(v)| d v\right)^{2} \frac{d w d t}{t^{2 n+1}} \\
& \leq C 2^{2 i n} \int_{0}^{\frac{|z|}{2^{i+1}}} t^{-4 n}\left(\frac{|z|}{t}\right)^{-(4 n+3)}\left(\frac{r}{t}\right)^{2} \frac{d t}{t} \leq C 2^{2 i n-i} \frac{r^{2}}{|z|^{4 n+2}} .
\end{aligned}
$$

By Lemma 4 again, we get

$$
\begin{aligned}
I_{2} & \leq C \int_{\frac{|z|}{2^{i+1}}}^{\infty} \int_{|z-w|<2^{i} t}\left(\int_{B(0, r)} t^{-2 n}\left(\frac{r}{t}\right)|a(v)| d v\right)^{2} \frac{d w d t}{t^{2 n+1}} \\
& \leq C 2^{2 i n} \int_{\frac{|z|}{2^{i+1}}}^{\infty} t^{-4 n}\left(\frac{r}{t}\right)^{2} \frac{d t}{t} \leq C 2^{i(6 n+2)} \frac{r^{2}}{|z|^{2(2 n+1)}} .
\end{aligned}
$$

Thus,

$$
\int_{|z| \geq 2^{i+2} r}\left|S_{L, 2^{i}}^{k} a(z)\right| d z \leq C 2^{3 i n+i} \int_{|z| \geq 2^{i+2} 2_{r}} \frac{r}{|z|^{2 n+1}} d z \leq C 2^{3 i n} .
$$

Therefore, when $\lambda>3$, we prove $\left\|g_{\lambda, k}^{*} a\right\|_{L^{1}} \leq C$. Then Theorem 2(c) is proved.

\section{Competing interests}

The authors declare that they have no competing interests.

\section{Authors' contributions}

All authors contributed equally to the writing of this paper. All authors read and approved the final manuscript.

\section{Acknowledgements}

This paper is supported by National Natural Science Foundation of China (11471018), the Beijing Natural Science Foundation (1142005).

Received: 2 February 2015 Accepted: 8 May 2015 Published online: 28 May 2015

\section{References}

1. Anderson, RF: The multiplicative Weyl functional calculus. J. Funct. Anal. 9, 423-440 (1972)

2. Grassman, A, Loupias, G, Stein, EM: An algebra of pseudo-differential operators and quantum mechanics in phase space. Ann. Inst. Fourier (Grenoble) 18, 343-368 (1969)

3. Peetre, J: The Weyl transform and Laguerre polynomials. Matematiche 27, 301-323 (1972)

4. Mauceri, G, Picardello, M, Ricci, F: A Hardy space associated with twisted convolution. Adv. Math. 39, $270-288$ (1981)

5. Mauceri, G, Picardello, M, Ricci, F: Twisted convolution, Hardy spaces and Hörmander multipliers. In: Proceedings of the Seminar on Harmonic Analysis (Pisa, 1980). Rend. Circ. Mat. Palermo (2), pp. 191-202 (1981)

6. Mauceri, G: The Weyl transform and bounded operators on $L^{p}\left(\mathbb{R}^{n}\right)$. J. Funct. Anal. 39, 408-429 (1980)

7. Huang, J, Wang, J: $\mathcal{H}^{p}$-Boundedness of Weyl multiplier. J. Inequal. Appl. 2014, 422 (2014)

8. Huang, J: Some characterizations of Hardy space associated with twisted convolution. Bull. Aust. Math. Soc. 79 405-417 (2009) 
9. Huang, J: The boundedness of Weyl multiplier on Hardy space associated with twisted convolution. Bull. Sci. Math. $133,588-596$ (2009)

10. Huang, J, Liu, Y: The molecular characterization of Hardy space associated with twisted convolution. J. Funct. Spaces 2014, 1-6 (2014)

11. Thangavelu, S: Lectures on Hermite and Laguerre Expansions. Math. Notes, vol. 42. Princeton University Press, Princeton (1993)

12. Coifman, RR, Meyer, Y, Stein, EM: Some new function spaces and their applications to harmonic analysis. J. Funct. Anal. 62, 304-335 (1985)

13. Stein, EM: Singular Integrals and Differentiability Properties of Functions. Princeton University Press, Princeton (1970)

14. Stein, EM: Topics in Harmonic Analysis Related to the Littlewood-Paley Theory. Annals of Mathematics Studies. Princeton University Press, Princeton (1970)

15. Auscher, P, Russ, E: Hardy spaces and divergence operators on strongly Lipschitz domain of $\mathbb{R}^{n}$. J. Funct. Anal. 201 148-184 (2003)

16. Folland, GB, Stein, EM: Hardy Spaces on Homogeneous Groups. Princeton University Press, Princeton (1982)

Submit your manuscript to a SpringerOpen ${ }^{\circ}$ journal and benefit from:

- Convenient online submission

- Rigorous peer review

- Immediate publication on acceptance

- Open access: articles freely available online

- High visibility within the field

- Retaining the copyright to your article 